\title{
Rol de la Neuroglia en el Trastorno del Espectro Autista
}

\author{
Role of Neuroglia in Autism Spectrum Disorder
}

Bélgica Vásquez ${ }^{1} \&$ Mariano del Sol $^{2}$

\begin{abstract}
VÁSQUEZ, B. \& DEL SOL, M. Rol de la neuroglia en el trastorno del espectro autista. Int. J. Morphol., 39(3):920-927, 2021.
RESUMEN: El trastorno del espectro autista (TEA) se caracteriza por presentar déficits persistentes en la comunicación y en la interacción social. Además, patrones de comportamiento, intereses o actividades de tipo restrictivo o repetitivo. Su etiología es compleja y heterogenia, y los mecanismos neurobiológicos que dan lugar al fenotipo clínico aún no se conocen por completo. Las investigaciones apuntan a factores genéticos y ambientales que afectan el cerebro en desarrollo. Estos avances coinciden con un aumento en la comprensión de las funciones fisiológicas y el potencial patológico de la neuroglia en el sistema nervioso central (SNC) que llevó a la noción de la contribución fundamental de estas células en el TEA. Así, el objetivo de este artículo fue revisar brevemente los factores de riesgo clave asociados al TEA y luego, explorar la contribución de la neuroglia en este trastorno. Se destaca el rol de los astrocitos, los microglocitos y los oligodendrocitos en el control homeostático del SNC, en la regulación inmunitaria del cerebro y en la mielinización axonal, así como el mal funcionamiento y las alteraciones morfológicas de estas células en los cerebros autistas.
\end{abstract}

PALABRAS CLAVE: Trastorno del espectro autista; Astrocito; Microgliocito; Oligodendrocito; Neuroglia.

\section{INTRODUCCIÓN}

El trastorno del espectro autista (TEA) se caracteriza por presentar déficits persistentes en la comunicación y en la interacción social. Además, patrones repetitivos y restringidos de conducta, actividades e intereses (APA, 2013). Los síntomas del TEA se manifiestan en la infancia temprana y su gravedad y presentación varían considerablemente entre los individuos, lo que da lugar a la clasificación de "espectro". Debido a un mayor diagnóstico, la prevalencia del TEA ha aumentado a lo largo de los años, con informes recientes que sugieren que 1 de cada 59 niños tienen TEA en los Estados Unidos (Baio et al., 2018). El TEA se diagnostica con mayor frecuencia en hombres que en mujeres, con proporciones que se estimaban de 4:1 (Scott et al., 2002). Sin embargo, estudios más recientes señalan que la proporción esta más cerca de 3:1, ya que parece haber un sesgo de género en el diagnóstico, lo que significa que las niñas que cumplen con los criterios de TEA tienen un riesgo desproporcionado de no recibir un diagnóstico clínico (Loomes et al., 2017).

La etiología del autismo es compleja y heterogenia, y los mecanismos neurobiológicos que dan lugar al fenotipo clínico aún no se conocen por completo. Las investigaciones apuntan a factores genéticos y ambientales aislados o asociados. La mayoría de estos factores convergen en alteraciones en la sinaptogénesis y función sináptica (Guang et al., 2018), en las respuestas inmunes durante el desarrollo prenatal o postnatal temprano (Estes \& McAllister, 2015) y en la conectividad neuronal en el cerebro en desarrollo (Stamou et al., 2013).

Un área importante de la investigación intenta comprender si estas anomalías convergen en un mecanismo común en el cerebro de personas con TEA que dan como resultado el fenotipo clínico compartido. Bajo este contexto, los avances coincidieron con un aumento notable en la comprensión de las funciones fisiológicas y el potencial patológico de la neuroglia en el sistema nervioso central (SNC) que llevó a una noción de la contribución fundamental de las células neurogliales en el TEA. Con base en lo anterior, este artículo tiene por objetivo revisar brevemente los factores de riesgo clave asociados al TEA y luego, explorar la contribución de la patología celular de la neuroglia en este trastorno.

\section{Factores de riesgo asociados al trastorno del espectro autista}

La heterogeneidad genética del TEA ha dificultado la identificación de genes específicos asociados con el trastorno, lo que ha obstaculizado los esfuerzos por develar los mecanismos involucrados (State \& Levitt, 2011). Los genes

\footnotetext{
${ }^{1}$ Facultad de Ciencias de la Salud, Universidad de Tarapacá, Iquique, Chile.

${ }^{2}$ Centro de Excelencia en Estudios Morfológicos y Quirúrgicos (CEMyQ), Universidad de La Frontera, Temuco, Chile.
} 
que se han relacionado con el TEA se pueden agrupar en tres categorías amplias: los que participan en la estructura y actividad de las sinapsis (Etherton et al., 2011a, b; Peca \& Feng, 2012), los que participan en la síntesis de proteínas (Kelleher \& Bear, 2008) y los implicados en la regulación de la expresión génica (van Bokhoven, 2011). Muchos de ellos codifican proteínas que tienen una función sináptica clara, por lo que se les atribuye un rol neurocéntrico en el TEA, en la cual se ven afectadas, directa o indirectamente, la sinaptogénesis, la estructura y función de las neuronas y la conectividad funcional local y de largo alcance en el SNC (Jan \& Jan, 2010; Peca \& Feng; Bourgeron, 2015; Long et al., 2016; O'Reilly et al., 2017; Martínez-Cerdeño et al., 2017; Joensuu et al., 2018; Guang et al.; Falougy et al., 2019).

Aunque la participación de anomalías genéticas en el autismo está bien aceptada, estudios recientes han indicado que existe una contribución importante de los factores ambientales no heredados que actúan de manera sinérgica o en paralelo en el cerebro aumentando la probabilidad de desarrollar TEA (Hallmayer et al., 2011; Estes \& McAllister). Además de la exposición a sustancias químicas o toxinas, estos incluyen factores como la edad de los padres en el momento de la concepción, la nutrición y la desregulación inmunológica materna durante la gestación y post natal del individuo (Grabrucker, 2012).

Numerosos factores interrelacionados pueden conducir a la desregulación del sistema inmunológico materno, por ejemplo, infecciones durante el embarazo por virus como el de la rubéola (Hutton, 2016) o infecciones bacterianas (Zerbo et al., 2015), creando un entorno inmunológico inflamatorio y estimulando la producción de citocinas maternas que pueden atravesar la placenta y provocar efectos duraderos sobre el desarrollo del feto, contribuyendo a los déficits del desarrollo neurológico observados en el TEA (Meltzer \& Van de Water., 2017). Además, un subconjunto de mujeres puede producir autoanticuerpos anti-cerebrales que también pueden acceder al cerebro fetal y actuar contra proteínas fetales aumentando el riesgo de desarrollar TEA (Braunschweig et al., 2013).

Se ha observado que la desregulación inmune continúa persistiendo en el TEA. Después del nacimiento y al menos durante la infancia, un individuo con TEA puede tener autoanticuerpos anti-cerebrales endógenos, separados de cualquier IgG materna; estos autoanticuerpos se han correlacionado con comportamientos alterados y desarrollo deficiente del sistema nervioso (Goines et al., 2011; Piras et al., 2014). También existe un panorama amplio de la desregulación inmunitaria relacionada con el TEA, que incluye múltiples redes del sistema inmunológico, como las vías que regulan las citocinas y las células NK, que juntas constituyen un entorno endógeno de respuesta y regulación inmunitaria atípica (Meltzer \& Van de Water).

\section{Rol de la neuroglia en la homeostasis y sistema defensi- vo en el tejido neuronal}

La función común de las células neurogliales es mantener la homeostasis del SNC. Con el desarrollo de herramientas electrofisiológicas, moleculares y genéticas, ahora está bien aceptado que los astrocitos representan una población diversa de células con numerosas funciones. Una de las funciones aceptadas de los astrocitos es la de mantener un entorno viable del sistema nervioso para las neuronas. Esto incluye amortiguar el exceso de potasio y de neurotransmisores, proporcionar nutrientes y soporte estructural alrededor de las sinapsis y contribuir a la integridad de la barrera hematoencefálica. También se sabe que los astrocitos liberan moléculas importantes, como el glutamato y la glutamina precursora de GABA, además de secretar captadores de especies reactivas de oxígeno (ROS) para la supervivencia neuronal. Por otra parte, los astrocitos que expresan la proteína ácida fibrilar glial (GFAP) actúan como células madres neuronales en las zonas neurogénicas adultas de la zona subventricular y subgranular del giro dentado del hipocampo (para una descripción completa de las funciones de los astrocitos ver también Wang \& Bordey, 2008; Parpura \& Verkhratsky, 2012; Haydon \& Nedergaard, 2014; Perez-Catalan et al., 2021).

Los microgliocitos son células inmunitarias residentes en el SNC (Graeber, 2010) que comparten muchas, si no todas, las propiedades de los macrófagos en otros tejidos y, en consecuencia, desempeñan un papel importante en las infecciones e inflamación del cerebro. Estudios in vivo de imágenes han revelado que, en el cerebro sano en reposo, los microgliocitos son muy dinámicos y se mueven constantemente para examinar activamente el parénquima cerebral. Estas células activas pueden responder rápidamente a agresiones patológicas, activándose para producir una serie de productos secretores que se cree que contribuyen a la defensa $y$, potencialmente, a dañar el cerebro infectado (Kettenmann et al., 2011). Por otra parte, se está reconociendo que las interacciones entre los microgliocitos y las neuronas son importantes para dar forma a la actividad del circuito neural en condiciones fisiológicas normales. Durante el desarrollo y la neurogénesis, las interacciones de los microgliocitos con las neuronas ayudan a dar forma a los patrones finales de los circuitos neuronales importantes para el comportamiento y con implicaciones para las enfermedades. Algunas de sus funciones fisiológicas son la fagocitosis de neuronas en desarrollo (Marín-Teva et al., 2004) y nacidas de adultos (Sierra et al., 2010; Diaz-Aparicio et al., 2020); monitorear las sinapsis con extensión y retrac- 
ción dinámicas de sus procesos celulares altamente ramificados (Tremblay et al., 2010; Wake et al., 2009); y fagocitosis de sinapsis durante el desarrollo sináptico normal (Paolicelli et al., 2011; Schafer et al., 2012) (para una descripción más completa de las funciones de los microgliocitos ver también Rock et al., 2004; Tremblay et al., 2011; Wake \& Fields, 2011; Wake et al., 2011).

Otra clase de célula neuroglial son los oligodendrocitos, los cuales son críticos para la mielinización del axón en el SNC. Son el producto final de un linaje celular que debe someterse a un programa complejo y cronometrado de proliferación, migración, diferenciación y mielinización para producir finalmente la vaina aislante de los axones (Bradl \& Lassmann, 2010). La evidencia actual indica que la vaina de mielina es más que una estructura de membrana aislante inerte. Los oligodendrocitos son metabólicamente activos y funcionalmente conectados al axón subyacente a través de canales mielínicos en el citoplasma para el movimiento de macromoléculas hacia y desde el espacio internodal periaxonal debajo de la vaina de mielina (Simons \& Nave, 2015). Además, se ha develado que los oligodendrocitos pueden mielinizar los axones de una manera dependiente de la actividad e influir en sus propiedades de velocidad de conducción (Gibson et al., 2014; Hines et al., 2015).

\section{Potencial patológico de la neuroglia en el trastorno del espectro autista}

La importancia de las células neurogliales en la fisiopatología del TEA, están respaldados por datos obtenidos de cerebro humano post mortem que muestran un aumento de gliosis reactiva y proliferación de células gliales (Morgan et al., 2012; Tetreaul et al., 2012; Edmonson et al., 2014) y por estudios de imágenes funcionales de tomografía por emisión de positrones (PET) que muestra la activación glial en múltiples regiones del cerebro de adultos jóvenes con TEA, como en el cerebelo, mesencéfalo, puente, giro fusiforme y corteza cingulada anterior y, orbitofrontal (Suzuki et al., 2013).

Considerando las múltiples funciones que desarrollan las células neurogliales en el sistema nervioso, es plausible pensar que las alteraciones funcionales de estas células podrían provocar déficit neurológico. De hecho, las enfermedades neurológicas son, por definición, fallas de la homeostasis del sistema nervioso en respuesta a factores endógenos o ambientales. Bajo esta línea, las células neurogliales, que son el elemento central de la homeostasis cerebral, participan en última instancia en la patogénesis de varios trastornos neurológicos incluidos el TEA (Giaume $e t$ al., 2007; Verkhratsky et al., 2013; Kim et al., 2020).
Los cambios patológicos de la neuroglia son complejos y se pueden clasificar en respuestas reactiva (astrogliosis, activación de los microgliocitos y remodelación walleriana de oligodendrocitos), degeneración con atrofia y pérdida de función (características de astrocitos y microgliocitos) y, remodelación patológica (Pekny et al., 2016; Coomey et al., 2020; Galvez-Contreras et al., 2020).

El análisis transcriptómico de los cerebros de pacientes con TEA indicó una asociación significativa de la patología con genes relacionados con la gliosis reactiva y la neuroinflamación (Voineagu et al., 2011). De acuerdo con esto, se han reportado altos niveles de varias citocinas proinflamatorias, como interleucina (IL) -6, TNF $\alpha$, IL-1ß, proteína quimioatrayente de macrófagos (MCP) -1 y factor de crecimiento tumoral (TGF)-1ß derivado de la neuroglia, en los tejidos cerebrales post mortem de personas con TEA (Vargas et al., 2005; Li et al., 2009; Wei et al., 2011). Los niveles muy elevados de expresión de IL$1 \beta$ afectan la proliferación de células progenitoras neurales en el SNC y contribuyen al crecimiento cerebral desviado específico de la región en pacientes con TEA ( $\mathrm{Li}$ et al.). IL- $1 \beta$ aumenta el nivel de expresión de IL- 6 y TNF- $\alpha$, que contribuyen a la inducción de la apoptosis de las células neuronales (Widera et al., 2006). La IL-6 puede modular comportamientos similares al autismo a través de deficiencias en la formación de sinapsis, el desarrollo de la columna dendrítica y el equilibrio del circuito neuronal (Wei et al.; 2011, 2012).

En la actualidad se reconoce que los factores proinflamatorios juegan un rol importante en la etiología del TEA, cuyo inicio patológico ocurre durante el desarrollo temprano del cerebro (Estes \& McAllister; Gupta et al., 2014; Voineagu et al.). Las citocinas proinflamatorias en el cerebro en desarrollo conducen clásicamente a neuroinflamación, una condición en la que los astrocitos y los microgliocitos se vuelven reactivos (gliosis), proliferan $\mathrm{y}$, dependiendo de la entidad de la gliosis, reclutan leucocitos periféricos y así amplifican el daño tisular inicial (Sofroniew, 2015).

La gliosis reactiva puede exacerbar las condiciones inflamatorias causadas por la activación inmune y contribuir a la patogénesis del TEA, sin embargo, aún no está claro los mecanismos moleculares y celulares subyacentes al TEA. Al respecto estudios han demostrado que la gliosis reactiva puede alterar la capacidad de los microgliocitos y los astrocitos para modular la maduración, eliminación (fagocitosis) o funcionamiento de las sinapsis en desarrollo porque las redes neuronales en desarrollo son altamente vulnerables a las agresiones que afectan las vías gliales que gobiernan la poda sináptica (Chung et al., 2015). 
Por otra parte, y como es sabido, las personas con TEA muestran un patrón de crecimiento alterado en varias regiones de la sustancia blanca que varía a lo largo de la vida (Courchesne et al., 2001, 2003). Algunas de estas anomalías neuroanatómicas se han asociado con cambios extensos en la mielinización y estrés oxidativo (Chauhan \& Chauhan, 2006)

El patrón de mielinización neuronal atípico por parte de los oligodendricitos se ha relacionado con varios factores, incluida la alteración genética y los factores epigenéticos. Algunos genes y factores de crecimiento implicados en este proceso de mielinización anormal incluyen Olig1, Olig2, Sox10, PGFRA, Nkx2-2, Gpr17, IGF-1 y EGF (Galvez-Contreta, et al.).

Por otro lado, se ha demostrado que la neuroinflamación y las citocinas proinflamatorias liberadas por los microgliocitos pueden alterar el estrés oxidativo (Pangrazzi et al., 2020; Kim et al.). El estrés oxidativo también se ha implicado en la fisiopatología del TEA al afectar el proceso de mielinización (Yui et al., 2016). En este sentido, los radicales oxidantes pueden dañar la población de oligodendrocitos que no logra diferenciarse en oligodendrocitos maduros formadores de mielina y aumentar una proliferación significativa de células precursoras de oligodendrocitos (OPC) que, a su vez, alteran todo el proceso de mielinización (Back et al., 2017). Los oligodendrocitos son un linaje celular muy susceptible a los radicales oxidantes porque tienen niveles bajos de glutatión (una molécula antioxidante altamente eficaz) y cantidades elevadas de esfingolípidos (Thorburne \& Juurlink, 1996; McTigue \& Tripathi, 2008).

\section{Morfología neuroglial en el trastorno del espectro autista}

Los estudios morfológicos de las células neurogliales en el autismo pueden contribuir notablemente a la comprensión de la fisiopatología del TEA, ya que las alteraciones morfológicas de las células neurogliales en la corteza autista puede afectar críticamente la neurogénesis, la función sináptica y neuronal, la plasticidad y la homeostasis de ciertas moléculas como el glutamato, lo que puede conducir al desarrollo de comportamientos autistas (Vargas et al.; Lee et al., 2017).

Los astrocitos son posiblemente las células neurogliales más diversas, y están representadas principalmente por los astrocitos protoplásmicos de la sustancia gris y los astrocitos fibrosos de la sustancia blanca. Su morfología, recuerda a una estrella por la gran cantidad de prolongaciones que irradian del soma hacia células vecinas. Estas prolongaciones, llamadas gliofilamentos, están ma- yormente compuestas por GFAP, específica para los astrocitos del SNC y que es comúnmente empleada como proteína marcadora. El núcleo de los astrocitos es más claro que el de otras células de la glía y el citoplasma contiene numerosos gránulos de glucógeno (Kimelberg, 2004). La generación y expansión de los astrocitos se completa en gran medida antes del nacimiento, pero la elaboración y maduración de sus finos procesos perisinápticos persiste durante el período activo de sinaptogénesis en el período posnatal (Ullian et al., 2001). Lo anterior sugiere que estas células están en una posición crucial para comunicarse activamente con las neuronas durante la sinaptogénesis y, por tanto, coordinar el desarrollo de los circuitos neuronales (Petrelli et al., 2016).

Los insultos de diferente gravedad y etiología inducen distintos programas astroglióticos clasificados como astrogliosis isomórfica (es decir, conservando la morfología y generalmente completamente reversible) y anisomórfica (es decir, cambiando la morfología, en la que los astrocitos pierden su organización de dominio y forman una cicatriz gliótica). Los astrocitos reactivos se caracterizan por una expresión de alto nivel de GFAP y por la regulación positiva de los filamentos intermedios en el citoplasma (Wilhelmsson et al., 2006).

Existen evidencias de activación alterada de astrocitos en el cerebelo, corteza frontal y cingulada en cerebros autistas (Fatemi et al., 2002; Laurence \& Fatemi, 2005; Vargas et al.), lo que se suma al peso de la evidencia patológica a favor de la desregulación inmunológica en el TEA. También se ha identificado una mayor expresión de GFAP en múltiples regiones del SNC en personas autistas, como cerebelo, giros frontal superior y medio, corteza cingulada anterior y corteza parietal (Laurence \& Fatemi; Vargas et al.; Fatemi et al., 2008; Voineagu et al.). En contraste con el aumento de los niveles de GFAP dentro del cerebro en el autismo, Morgan et al. (2014) reportaron ausencia de alteraciones significativas en cuanto al número y tamaño de los astrocitos en la amígdala de cerebros post mortem de individuos varones adolescentes y adultos con TEA. Señalaron, sin embargo, que la ausencia de alteraciones no indicaría necesariamente que no exista activación de los astrocitos en el TEA, ya que los astrocitos no proliferan significativamente cuando se activan. Bajo esta misma línea, Lee et al. tampoco encontraron evidencias de alteración en la densidad, tamaño del soma y longitud de los procesos piales en la sustancia blanca de la corteza prefrontal dorso lateral en individuos autistas. Señalaron que la falta de evidencia podría deberse al reducido tamaño de la muestra y a un amplio rango de edad $(\mathrm{n}=8$, edad $=$ 4-51 años), con características patológicas potencialmente restringidas a las primeras etapas del autismo. 
Los microgliocitos son células pequeñas con poco citoplasma y un número variable de proyecciones celulares cortas e irregulares denominadas procesos microgliales. La forma del núcleo de la célula microglial es muy variable, con núcleos esféricos, elípticos, de forma irregular y triangulares (Morgan et al., 2014). Estas células pueden sufrir cambios estructurales notables en función de la localización exacta en el SNC y de las necesidades concretas del organismo. Presentan tres fases morfológicas que reflejan su plasticidad: una fase ameboide que se encuentra en el feto, una fase ramificada (de reposo) en la cual los procesos microgliales se extienden y retraen constantemente para controlar el entorno local y una tercera fase ameboide de recuperación de propiedades y motilidad, necesarias para el desempeño activo de su función macrofágica (Rock et al.).

Se ha informado activación microglial, a través de imágenes in vivo (PET), en varias regiones cerebrales, como el mesencéfalo, el puente, los giros fusiformes, las cortezas cingulada anterior y orbitofrontal y el cerebelo en personas con TEA (Suzuki et al.). Así mismo, se ha informado activación microglial en tejido post mortem de la corteza prefrontal dorso lateral en algunas personas autistas, con alteraciones morfológicas que incluyen agrandamiento del soma, retracción y engrosamiento de los procesos microgliales y extensión de los filopodios; además, aumento significativo del promedio de somas microgliales en la sustancia blanca, con una tendencia similar en la sustancia gris (Morgan et al., 2010). También, se ha demostrado mayor densidad microglial en la sustancia gris del cerebelo (Vargas et al.), la corteza frontoinsular y visual (Tetreault $e t$ al., 1996) en personas con TEA. En algunas regiones del cerebro autista (por ejemplo, corteza prefrontal dorso lateral), se ha observado un aumento de las interacciones microglía-neurona a corta distancia (Morgan et al., 2012).

En contraste con lo anterior, Morgan et al. (2014) reportaron que en general, el número y el volumen promedio de los microgliocitos en la amígdala no diferían entre los TEA y los cerebros en desarrollo típico. Sin embargo, observaron heterogeneidad evidente dentro de la cohorte de los TEA. Dos de los ocho cerebros con TEA mostraron una fuerte activación microglial. Los autores señalaron que los microgliocitos en reposo presentaban cuerpos celulares pequeños y esféricos, y los procesos eran delgados y bien definidos. Los microgliocitos levemente activados mostraban procesos ligeramente engrosados, pero relativamente rectos, con algunas pequeñas zonas hinchadas. Los microgliocitos moderadamente activados presentaban cuerpos celulares notablemente agrandados, con procesos tortuosos gruesos y desigualmente hinchados. Los microgliocitos fuertemente activados presentaban cuerpos celulares más agrandados y una pérdida de procesos; los procesos restantes eran cortos y gruesos, ya que las células se aproximaban a una morfología ameboide. Finalmente, los microgliocitos extremadamente activados mostraban cuerpos celulares aún más grandes y pocos o ningún proceso, asumiendo en gran medida una morfología ameboide.

Los oligodendrocitos son la población glial más numerosa del cerebro. Presentan pequeños cuerpos celulares llenos de núcleos y menor cantidad de prolongaciones que los astrocitos. Sus prolongaciones carecen de fibras, pero se encuentran llenas de gránulos citoplasmáticos (Simons \& Nave). Los núcleos de los oligodendrocitos suelen tener un diámetro de $2,5 \mu \mathrm{m}$ a $5 \mu \mathrm{m}$. Tienen una alta densidad de gránulos grandes y oscuros de cromatina (típicamente 5-15), con una baja densidad de gránulos más pequeños y ligeros en comparación con otros núcleos gliales. Se tiñen de manera más oscura con $\mathrm{H} \& \mathrm{E}$ que cualquier otro tipo de núcleo; tienen un límite nuclear oscuro y son principalmente de forma esférica u ovalada (Morgan et al., 2014).

Los oligodendrocitos satélites (perineuronales) se encuentran próximos a los cuerpos neuronales en la sustancia gris y a los vasos sanguíneos y no forman mielina. Los oligodendrocitos interfasciculares se encuentran sobre todo en la sustancia blanca (Simons \& Nave). Estos últimos, del Río-Hortega (1921) los clasificó en cuatro tipos: las células de tipo I, que generan muchos segmentos de mielina diferentes en axones de pequeño diámetro en diversas orientaciones; las células de tipo II que son similares a las de tipo I en tamaño y número, pero los segmentos de mielina corren en paralelo entre sí; los oligodendrocitos de tipo III que envuelven menos axones de mayor diámetro; y los oligodendrocitos de tipo IV que tienen un cuerpo celular muy próximo a un único axón muy grande, similar a los schwanocitos (neurolemocitos).

En cuanto a los oligodendrocitos y su rol en el TEA, existen pocas evidencias de anomalías morfológicas. Un estudio piloto en niños de entre 4 y 6 años con diagnóstico de autismo mostró que el crecimiento desmedido de las regiones frontal y temporal podría deberse a una mielinización excesiva (Carmody \& Lewis, 2010). Los autores señalaron que estos cambios parecen ser específicos del área del cerebro, ya que la mielinización en la corteza medial izquierda y derecha fue mayor a la esperada para su edad en niños con TEA; pero al mismo tiempo, menor de la esperada en la unión temporoparietal izquierda.

Evidencias adicionales en adolescentes indican que los cerebros con TEA muestran menos axones, menos volumen axonal y una baja densidad de tractos en el cuerpo calloso, fascículo frontal-occipital, fascículo uncinado derecho y fascículo arqueado derecho (Dimond et al., 2019). Al 
parecer, los cambios patológicos en la citoarquitectura de la sustancia blanca en todos los lóbulos del cerebro (Herbert et al., 2004) y la tasa de mielinización alterada en el cuerpo calloso (Parellada et al., 2014) pueden explicar la conectividad disfuncional encontrada en las regiones parietal medial y temporoparietal (Courchesne et al., 2005).

Los adultos con TEA muestran un número significativamente menor de oligodendrocitos en la amígdala en comparación con los controles de desarrollo típico de la misma edad. (Morgan et al., 2014). Una pérdida de oligodendrocitos podría tener un impacto significativo en la conectividad funcional de la amígdala, lo que podría generar importantes dificultades cognitivas para las personas mayores con TEA (Arnett et al., 2001; Monk et al., 2010).

\section{CONCLUSIONES}

Debido a que el TEA presenta una etiología compleja y heterogénea, es crucial definir e investigar los distintos actores causantes de esta condición. Las evidencias demuestran alteraciones funcionales y morfológicas de las células neuroglial en el cerebro autista, lo que afectar críticamente la función sináptica y neuronal, la homeostasis del SNC, la respuesta inmune y la conectividad neuronal. En base a lo anterior, los astrocitos, microgliocitos y oligodendrocitos podrían considerarse dianas para nuevas estrategias terapéuticas.

VÁSQUEZ, B. \& DEL SOL, M. Role of neuroglia in autism spectrum disorder. Int. J. Morphol., 39(3):920-927, 2021.

SUMMARY: Autism spectrum disorder (ASD) is characterized by persistent deficits in communication and social interaction, as well as restrictive or repetitive activities or interests. Its etiology is complex and heterogeneous, and the neurobiological mechanisms that give rise to the clinical phenotype are not yet fully understood. Research points to genetic and environmental factors that affect the developing brain. These advances are consistent with an enhanced understanding of the physiological functions and pathological potential of neuroglia in the central nervous system (CNS) which supports the conclusion of the contribution of these cells in ASD. Therefore, the objective of this article was to briefly review the key risk factors associated with ASD and then explore the contribution of glia in this disorder. The role of astrocytes, microgliocytes and oligodendrocytes in the homeostatic control of the CNS in the immune regulation of the brain and in axonal myelination, as well as malfunction and morphological alterations of these cells in autistic brains are emphasized.

KEY WORDS: Autism spectrum disorder; Astrocyte; Microgliocyte; Ologodendrocyte; Neuroglia.

\section{REFERENCIAS BIBLIOGRÁFICAS}

American Psychiatric Association (APA). Diagnostic and statistical manual of mental disorders. $5^{\text {th }}$ ed. Washington DC, 2013.

Arnett, H. A.; Mason, J.; Marino, M.; Suzuki, K.; Matsushima, G. K. \& Ting, J. P. TNF alpha promotes proliferation of oligodendrocyte progenitors and remyelination. Nat. Neurosci., 4(11):1116-22, 2001.

Back, S. A. White matter injury in the preterm infant: Pathology and mechanisms. Acta Neuropathol., 134(3):331-49, 2017.

Baio, J.; Wiggins, L.; Christensen, D. L.; Maenner, M. J.; Daniels, J.; Warren, Z.; Kurzius-Spencer, M.; Zahorodny, W.; Robinson Rosenberg, C.; White, T.; Durkin, M. S.; Imm, P.; Nikolaou, L.; Yeargin-Allsopp, M.; Lee, L. C.; Harrington, R. ;Lopez, M.; Fitzgerald, R. T.; Hewitt, A.; Pettygrove, S.; Constantino, J. N.; Vehorn, A.; Shenouda, J.; Hall-Lande, J.; Van Naarden Braun, K. \& Dowling, N. F. Prevalence of autism spectrum disorder among children aged 8 years - Autism and developmental disabilities monitoring network, 11 Sites, United States, 2014. MMWR Surveill Summ., 67(6):1-23, 2018.

Bourgeron, T. From the genetic architecture to synaptic plasticity in autism spectrum disorder. Nat. Rev. Neurosci., 16(9):551-63, 2015.

Bradl, M. \& Lassmann, H. Oligodendrocitos: biología y patología. Acta Neuropathol., 119(1):37-53, 2010.

Braunschweig, D.; Krakowiak, P.; Duncanson, P.; Boyce, R.; Hansen, R. L.; Ashwood, P.; Hertz-Picciotto, I.; Pessah, I. \& Van de Water, J. Autism-specific maternal autoantibodies recognize critical proteins in developing brain. Transl. Psychiatry., 3:e277, 2013.

Carmody, D.P. \& Lewis, M. Regional white matter development in children with autism spectrum disorders. Dev. Psychobiol., 52(8):755- 63, 2010.

Chauhan, A. \& Chauhan, V. Oxidative stress in autism. Pathophysiology, 13(3):171-81, 2006.

Coomey, R.; Stowell, R.; Majewska, A. \& Tropea, D. The Role of Microglia in Neurodevelopmental Disorders and their Therapeutics. Curr. Top Med. Chem., 20(4):272-6, 2020.

Courchesne, E.; Karns, C. M.; Davis, H. R.; Ziccardi, R.; Carper, R. A.; Tigue, Z. D.; Chisum, H. J.; Moses, P.; Pierce, K.; Lord, C.; Lincoln, A. J.; Pizzo, S.; Schreibman, L.; Haas, R. H.; Akshoomoff, N. A. \& Courchesne, R. Y. Unusual brain growth patterns in early life in patients with autistic disorder: An MRI study. Neurology, 57(2):245-54, 2001.

Courchesne, E.; Carper, R.\& Akshoomoff, N. Evidence of brain overgrowth in the first year of life in autism. J. Am. Med. Assoc., 290(3):337-44, 2003.

Courchesne, E. \& Pierce, K. Why the frontal cortex in autism might be talking only to itself: Local over-connectivity but long-distance disconnection. Curr. Opin. Neurobiol., 15(2):225-30, 2005.

Chung. W; Choi, S. Y.; Lee, E.; Park, H.; Kang, J.; Park, H.; Choi, Y.; Lee, D.; Park, S. G.; Kim, R.; Cho, Y. S.; Choi, J.; Kim, M. H.; Lee, J. W.; Lee, S.; Rhim, I.; Jung, M. W.; Kim, D.; Bae, Y. C. \& Kim, E. Social deficits in IRSp 53 mutant mice improved by NMDAR and mGluR5 suppression. Nat. Neurosci., 18(3):435-43, 2015.

Del Río-Hortega P. La glía de escasas radiaciones (oligodendroglía). Bol. Real Soc. Esp. Hist. Nat., 21:63-92, 1921.

Diaz-Aparicio, I.; Paris, I.; Sierra-Torre, V.; Plaza-Zabala, A.; RodríguezIglesias, N.; Márquez-Ropero, M.; Beccari, S.; Huguet, P.; Abiega, O.; Alberdi, E.; Matute, C.; Bernales, I.; Schulz, A.; Otrokocsi, L.; Sperlagh, B.; Happonen, K. E.; Lemke, G.; Maletic-Savatic, M.; Valero, J. \& Sierra, A. Microglia actively remodel adult hippocampal neurogenesis through the phagocytosis secretome. J. Neurosci., 40(7):1453-82, 2020.

Dimond, D.; Schuetze, M.; Smith, R. E.; Dhollander, T.; Cho, I.; Vinette, S.; Ten Eycke, K.; Lebel, C.; McCrimmon, A.; Dewey, D.; Connelly, A. \& Bray, S. Reduced white matter fiber density in autism spectrum disorder. Cereb. Cortex, 29(4):1-11, 2019.

Edmonson, C.; Ziats, M. N. \& Rennert, O. M. Altered glial marker expression in autistic post-mortem prefrontal cortex and cerebellum. Mol. Autism, 5(1):3, 2014. 
Estes, M. L. \& McAllister, A. K. Immune mediators in the brain and peripheral tissues in autism spectrum disorder. Nat. Rev. Neurosci., 16(8):469-86, 2015.

Etherton, M.; Földy, C.; Sharma, M.; Tabuchi, K.; Liu, X.; Shamloo, M.; Malenka, R. C. \& Südhof, T. C. Autism-linked neuroligin-3 R451C mutation differentially alters hippocampal and cortical synaptic function. Proc. Natl. Acad. Sci. U S A., 108(33):13764-9, 2011 a.

Etherton, M. R.; Tabuchi, K.; Sharma, M.; Ko, J. \& Südhof, T. C. An autism-associated point mutation in the neuroligin cytoplasmic tail selectively impairs AMPA receptor-mediated synaptic transmission in hippocampus. Embo. J., 30(14):2908-19, 2011b.

Falougy, H. E.; Filova, B.; Ostatnikova, D.; Bacova, Z. \& Bakos J. Neuronal morphology alterations in autism and possible role of oxytocin. Endocr. Regul., 53(1):46-54, 2019.

Fatemi, S. H.; Laurence, J.; Araghi-Niknam, M.; Stary, J. M. \& Rizvi, S. Glial fibrillary acidic protein is elevated in superior frontal and parietal cortices of autistic subjects. Int. J. Neuropsychopharmacol., 5:153-4, 2002.

Fatemi, S. H.; Folsom, T. D.; Reutiman, T. J. \& Lee, S. Expression of astrocytic markers aquaporin 4 and connexin 43 is altered in brains of subjects with autism. Synapse, 62(7):501-7, 2008.

Galvez-Contreras, A. Y.; Zarate-Lopez, D.; Torres-Chavez, A. L. \& Gonzalez-Perez, O. Role of oligodendrocytes and myelin in the pathophysiology of autism spectrum disorder. Brain Sci., 10(12):951, 2020.

Graeber, M. B. Changing face of microglia. Science, 330(6005):783-8, 2010.

Grabrucker, A. M. Environmental factors in autism. Front. Psychiatry, 3:118, 2012.

Giaume, C.; Kirchhoff, F.; Matute, C.; Reichenbach, A. \& Verkhratsky, A. Glia: the fulcrum of brain diseases. Cell Death Differ., 14(7):132435, 2007.

Gibson, E. M.; Purger, D.; Mount, C. W.; Goldstein, A. K.; Lin, G. L.; Wood, L. S.; Inema, I.; Miller, S.E.; Bieri, G.; Zuchero, J. B.; Barres, B. A.; Woo, P. J.; Vogel, H. \& Monje, M. Neuronal activity promotes oligodendrogenesis and adaptive myelination in the mammalian brain. Science, 344(6183):1252304, 2014.

Goines, P.; Haapanen, L.; Boyce, R.; Duncanson, P.; Braunschweig, D.; Delwiche, L.; Hansen, R.; Hertz-Picciotto, I.; Ashwood, P. \& Van de Water, J. Autoantibodies to cerebellum in children with autism associate with behavior. Brain Behav. Immun., 25(3):514-23, 2011.

Guang, S.; Pang, N.; Deng, X.; Yang, L.; He, F.; Wu, L.; Chen, C.; Yin, F. \& Peng, J. Synaptopathology involved in autism spectrum disorder. Front. Cell Neurosci., 12:470, 2018.

Gupta, S.; Ellis, S. E.; Ashar, F. N.; Moes, A.; Bader, J. S.; Zhan, J.; West, A. B. \& Arking, D. E. Transcriptome analysis reveals dysregulation of innate immune response genes and neuronal activity-dependent genes in autism. Nat. Commun., 5:5748, 2014.

Hallmayer, J.; Cleveland, S.; Torres, A.; Phillips, J.; Cohen, B.; Torigoe, T.; Miller, J.; Fedele, A.; Collins, J.; Smith, K.; Lotspeich, L.; Croen, L. A.; Ozonoff, S.; Lajonchere, C.; Grether, J. K. \& Risch, N. Genetic heritability and shared environmental factors among twin pairs with autism. Arch Gen Psychiatry., 68(11):1095-102, 2011.

Haydon, P. G. \& Nedergaard, M. How do astrocytes participate in neural plasticity? Cold Spring Harb Perspect Biol., 7(3):a020438, 2014.

Herbert, M. R.; Ziegler, D. A.; Makris, N.; Filipek, P. A.; Kemper, T. L.; Normandin, J. J.; Sanders, H. A.; Kennedy, D. N. \& Caviness, V.S. Localization of white matter volume increase in autism and developmental language disorder. Ann. Neurol., 55(4):530-40, 2004.

Hines, J. H.; Ravanelli, A. M.; Schwindt, R.; Scott, E. K. \& Appel, B. Neuronal activity biases axon selection for myelination in vivo. Nat. Neurosci., 18(5):683-9, 2015.

Hutton, J. Does rubella cause autism: A 2015 reappraisal? Front. Hum. Neurosci., 10:25, 2016.

Jan, Y. N. \& Jan, L. Y. Branching out: mechanisms of dendritic arborization. Nat. Rev. Neurosci., 11(5):316-28, 2010.
Joensuu, M.; Lanoue, V. \& Hotulainen, P. Dendritic spine actin cytoskeleton in autism spectrum disorder. Prog. Neuropsychopharmacol. Biol. Psychiatry., 84(Pt B):362-81, 2018.

Kelleher, R. J. \& Bear, M. F. The autistic neuron: troubled translation? Cell, 135(3):401-6, 2008

Kettenmann, H.; Hanisch, U. K.; Noda, M., \& Verkhratsky, A. Physiology of microglia. Physiol. Rev., 91(2):461-553, 2011.

Kim, Y. S.; Choi, J. \& Yoon, B. E. Neuron-Glia interactions in neurodevelopmental disorders. Cells, 9(10):2176, 2020.

Kimelberg, H. K. The problem of astrocyte identity. Neurochem. Int., 45(23):191-202, 2004

Laurence, J. A. \& Fatemi, S. H. Glial fibrillary acidic protein is elevated in superior frontal, parietal and cerebellar cortices of autistic subjects. Cerebellum, 4(3):206-10, 2005.

Lee, T. T.; Skafidas, E.; Dottori, M.; Zantomio, D.; Pantelis, C.; Everall, I. \& Chana, G. No preliminary evidence of differences in astrocyte density within the white matter of the dorsolateral prefrontal cortex in autism. Mol. autism, 8:64, 2017.

Li, X. H.; Chauhan, A.; Sheikh, A. M.; Patil, S.; Chauhan, V.; Li, X. M.; Ji, L.; Brown, T. \& Malik, M. Elevated immune response in the brain of autistic patients. J. Neuroimmunol., 207(1-2):111-6, 2009.

Long, Z.; Duan, X.; Mantini, D. \& Chen, H. Alteration of functional connectivity in autism spectrum disorder: effect of age and anatomical distance. Sci. Rep., 6:6527, 2016.

Loomes, R.; Hull, L. \& Mandy, W. P. L. What is the male-to-female ratio in autism spectrum disorder? A systematic review and meta-analysis. J. Am. Acad. Child. Adolesc. Psychiatry, 56(6):466-74, 2017.

Marín-Teva, J. L.; Dusart, I.; Colin, C.; Gervais, A.; Rooijen, N. \& Mallat, M. Microglia Promote the death of developing Purkinje cells. Neuron, 41(4):535-47, 2004.

Martínez-Cerdeño, V. Dendrite and spine modifications in autism and related neurodevelopmental disorders in patients and animal models. Dev. Neurobiol., 77(4):393-404, 2017.

McTigue, D. M.; Tripathi, R. B. The life, death, and replacement of oligodendrocytes in the adult CNS. J. Neurochem., 107(1):1-19, 2008.

Meltzer, A. \& Van de Water, J. The role of the immune system in autism spectrum disorder. Neuropsychopharmacol, 42(1):284-98, 2017.

Monk, C. S.; Weng, S. J.; Wiggins, J. L.; Kurapati, N.; Louro, H. M.; Carrasco, M.; Maslowsky, J.; Risi, S. \& Lord, C. Neural circuitry of emotional face processing in autism spectrum disorders. J. Psychiatry Neurosci., 35(2):105-14, 2010.

Morgan, J. T.; Chana, G.; Pardo, C. A.; Achim, C.; Semendeferi, K.; Buckwalter, J.; Courchesne, E. \& Everall, I. P. Microglial activation and increased microglial density observed in the dorsolateral prefrontal cortex in autism. Biol. Psychiatry, 68(4):368-76, 2010.

Morgan, J. T.; Chana, G.; Abramson, I.; Semendeferi, K.; Courchesne, E. \& Everall, I. P. Abnormal microglial-neuronal spatial organization in the dorsolateral prefrontal cortex in autism. Brain Res., 1456:72-81, 2012.

Morgan, J. T.; Barger, N.; Amaral, D. G. \& Schumann, C. M. Stereological study of amygdala glial populations in adolescents and adults with autism spectrum disorder. PLoS One, 9(10):e110356, 2014.

O'Reilly, C.; Lewis, J. D. \& Elsabbagh, M. Is functional brain connectivity atypical in autism? A systematic review of EEG and MEG studies. PLoS One, 12(5):e0175870, 2017.

Pangrazzi, L.; Balasco, L. \& Bozzi, Y. Oxidative stress and immune system dysfunction in autism spectrum disorders. Int. J. Mol. Sci., 21(9):3293, 2020.

Paolicelli, R. C.; Bolasco, G.; Pagani, F.; Maggi, L.; Scianni, M.; Panzanelli, P.; Giustetto, M.; Ferreira, T. A.; Guiducci, E.; Dumas, L.; Ragozzino, D. \& Gross, C. T. Synaptic pruning by microglia is necessary for normal brain development. Science, 333(6048):14568, 2011.

Parellada, M.; Penzol, M. J.; Pina, L.; Moreno, C.; González-Vioque, E.; Zalsman, G. \& Arango, C. The neurobiology of autism spectrum disorders. Eur. Psychiatry, 29(1):11-9, 2014. 
Parpura, V. \& Verkhratsky, A. Neuroglia at the crossroads of homoeostasis, metabolism and signalling: evolution of the concept. ASN Neuro, 4(4):201-5, 2012.

Peca, J. \& Feng, G. Cellular and synaptic network defects in autism. Curr. Opin. Neurobiol., 22(5):866-72, 2012.

Pekny, M.; Pekna, M.; Messing, A.; Steinhauser, C.; Lee, J. M.; Parpura, V.; Hol, E. M.; Sofroniew, M. V. \& Verkhratsky, A. Astrocytes: a central element in neurological diseases. Acta Neuropathol., 131(3):32345, 2016.

Perez-Catalan, N. A.; Doe, C. Q. \& Ackerman, S. D. The role of astrocytemediated plasticity in neural circuit development and function. Neural Dev., 16(1):1, 2021.

Petrelli, F.; Pucci, L. \& Bezzi, P. Astrocytes and microglia and their potential link with autism spectrum disorders. Front. Cell Neurosci., 10:21, 2016.

Piras, I. S.; Haapanen, L.; Napolioni, V.; Sacco, R.; Van de Water, J. \& Persico, A. M. Anti-brain antibodies are associated with more severe cognitive and behavioral profiles in Italian children with Autism Spectrum Disorder. Brain Behav. Immun., 38:91-9, 2014.

Rock, R. B.; Gekker, G.; Hu, S.; Sheng, W. S.; Cheeran, M.; Lokensgard, J. R. \& Peterson, P. K. Role of microglia in central nervous system infections. Clin. Microbiol. Rev., 17(4):942-64, 2004.

Schafer, D. P.; Lehrman, E. K.; Kautzman, A. G.; Koyama, R.; Mardinly, A. R.; Yamasaki, R.; Ransohff, R. M.; Greenberg, M. E.; Barres, B. A. \& Stevens, B. Microglia sculpt postnatal neural circuits in an activity and complement-dependent manner. Neuron, 74(4):691-705, 2012.

Scott, F. J.; Baron-Cohen, S.; Bolton, P. \& Brayne, C. Brief report: prevalence of autism spectrum conditions in children aged 5-11 years in Cambridgeshire, UK. Autism, 6(3):231-7, 2002.

Sierra, A.; Encinas, J. M.; Deudero, J. J.; Chancey, J. H.; Enikolopov, G.; Overstreet-Wadiche, L. S.; Tsirka, S. E. \& Maletic-Savatic, M. Microglia shape adult hippocampal neurogenesis through apoptosiscoupled phagocytosis. Cell Stem Cell, 7(4):483-95, 2010.

Simons, M. \& Nave, K. A. Oligodendrocytes: Myelination and axonal support. Cold Spring Harb. Perspect. Biol., 8(1):a020479, 2015.

Sofroniew, M. V. Astrocyte barriers to neurotoxic inflammation. Nat. Rev. Neurosci., 16(5):249-63, 2015.

Stamou, M.; Streifel, K. M.; Goines, P. E. \& Lein, P. J. Neuronal connectivity as a convergent target of gene $\times$ environment interactions that confer risk for Autism Spectrum Disorders. Neurotoxicol Teratol., 36:3-16, 2013.

State, M. W. \& Levitt, P. The conundrums of understanding genetic risks for autism spectrum disorders. Nat. Neurosci., 14(12):1499-506, 2011.

Suzuki, K.; Sugihara, G.; Ouchi, Y.; Nakamura, K.; Futatsubashi, M.; Takebayashi, K.; Yoshihara, Y.; Omata, K.; Matsumoto, K.; Tsuchiya, K. J.; Iwata, Y.; Tsujii, M.; Sugiyama, T. \& Mori, N. Microglial activation in young adults with autism spectrum disorder. JAMA Psychiatry, 70(1):49-58, 2013.

Tetreault, N. A.; Hakeem, A. Y.; Jiang, S.; Williams, B. A.; Allman, E.; Wold, B. J. \& Allman, J. M. Microglia in the cerebral cortex in autism. J. Autism Dev. Disord., 42(12):2569-84, 2012.

Tremblay, M. Ė.; Lowery, R. L. \& Majewska, A. K. Microglial interactions with synapses are modulated by visual experience. PLoS Biol., 8(11):e1000527, 2010.

Tremblay, M. Ė.; Stevens, B.; Sierra, A.; Wake, H.; Bessis, A. \& Nimmerjahn, A. The role of microglia in the healthy brain. J. Neurosci., 31(45):16064-9, 2011.

Thorburne, S. K. \& Juurlink, B. H. J. Low glutathione and high iron govern the susceptibility of oligodendroglial precursors to oxidative stress. J. Neurochem., 67(3):1014-22, 1996.

Ullian, E. M.; Sapperstein, S. K.; Christopherson, K. S. \& Barres, B. A. Control of synapse number by glia. Science, 29 (5504):657-61, 2001.

van Bokhoven, H. Genetic and epigenetic networks in intellectual disabilities. In: Annual review genetics, Vol. 45. Palo Alto, CA, Annual Reviews, 81-104, 2011.
Vargas, D. L.; Nascimbene, C.; Krishnan, C.; Zimmerman, A. W. \& Pardo, C. A. Neuroglial activation and neuroinflammation in the brain of patients with autism. Ann. Neurol., 57(1):67-81, 2005.

Verkhratsky, A. \& Butt, A. M. Glial Physiology and Pathophysiology. WileyBlackwell, 2013.

Voineagu, I.; Wang, X.; Johnston, P.; Lowe, J. K.; Tian, Y.; Horvath, S.; Mill, J..; Cantor, R. M.; Blencowe, B. J. \& Geschwind, D. H. Transcriptomic analysis of autistic brain reveals convergent molecular pathology. Nature., 474(7351):380-4, 2011.

Wake, H. \& Fields, R. D. Physiological function of microglia. Neuron Glia Biol., 7(1):1-3, 2011.

Wake, H.; Moorhouse, A. J.; Jinno, S.; Kohsaka, S. \& Nabekura J. Resting microglia directly monitor the functional state of synapses in vivo and determine the fate of ischemic terminals. J. Neurosci., 29(13):3974$80,2009$.

Wake, H.; Moorhouse, A. \& Nabekura, J. Functions of microglia in the central nervous system - beyond the immune response. Neuron Glia Biol., 7(1):47-53, 2011.

Wang, D. D. \& Bordey, A. The astrocyte odyssey. Prog. Neurobiol., 86(4):342-67, 2008.

Wei, H. G.; Zou, H.; Sheikh, A. M.; Malik, M.; Dobkin, C.; Brown, W. T. $\& \mathrm{Li}, \mathrm{X}$. IL-6 is increased in the cerebellum of autistic brain and alters neural cell adhesion, migration and synaptic formation. $J$. Neuroinflamm., 8:52, 2011.

Wei, H.; Chadman, K. K.; McCloskey, D. P.; Sheikh, A. M.; Malik, M.; Brown, W. T.; Li, X. Brain IL-6 elevation causes neuronal circuitry imbalances and mediates autism-like behaviors. Biochim. Biophys. Acta, 1822(6):831-42, 2012.

Widera, D.; Mikenberg, I.; Elvers, M.; Kaltschmidt, C.; Kaltschmidt, B. Tumor necrosis factor a triggers proliferation of adult neural stem cells via IKK/NF-kB signaling. BMC Neurosci., 7:64, 2006.

Wilhelmsson, U.; Bushong, E. A.; Price, D. L.; Smarr, B. L.; Phung, V.; Terada, M.; Ellisman, M. H. \& Pekny, M. Redefining the concept of reactive astrocytes as cells that remain within their unique domains upon reaction to injury. Proc. Natl. Acad. Sci. U.S.A., 103(46):175138, 2006.

Yui, K.; Kawasaki, Y.; Yamada, H.; Ogawa, S. Oxidative stress and nitric oxide in autism spectrum disorder and other neuropsychiatric disorders. CNS Neurol. Disord. Drug Targets, 15(5):587-96, 2016.

Zerbo, O.; Qian, Y.; Yoshida, C.; Grether, J. K.; Van de Water, J. \& Croen, L. A. Maternal infection during pregnancy and autism spectrum disorders. J. Autism Dev. Disord., 45(12):4015-25, 2015.

Zeidán-Chuliá, F.; Salmina, A. B.; Malinovskaya, N. A.; Noda, M.; Verkhratsky, A. \& Moreira, J. C. The glial perspective of autism spectrum disorders. Neurosci. Biobehav. Rev., 38:160-72, 2014.

\section{Dirección para correspondencia: \\ Dra. Bélgica Vásquez \\ Facultad de Ciencias de la Salud \\ Universidad de Tarapacá \\ Iquique \\ CHILE}

\section{Email: bvasquezp@uta.cl}

Recibido : 07-01-2021

Aceptado: 24-02-2021 